\title{
Assessing for Fetal Alcohol Spectrum Disorder: A National Survey of Assessment Measures Used by FASD Clinics in Canada
}

As a member of a Canadian clinic that provides asessment and diagnostic services for individuals with Fetal Alcohol Spectrum Disorder (FASD), you are invited to participate in this study.

Title: Assessing for Fetal Alcohol Spectrum Disorder: A National Survey of Assessment Measures Used by FASD Clinics in Canada

Investigators:

Kelly Coons-Harding, Laurentian University \& Canada FASD Research Network

Katherine Flannigan, Canada FASD Research Network

Colleen Burns, Lakeland Centre for FASD

Audrey McFarlane, Canada FASD Research Network \& Lakeland Centre for FASD

Kathy Unsworth, Canada FASD Research Network

What is this project about? The purpose of this project is to collect information about the testing measures used by FASD clinicians across Canada to evaluate neurodevelopmental functioning in their clients. The overall goals of the project are to:

- Provide an overview of the measuses used in Canada to assess for FASD across the lifespan

- Determine which measures are most commonly used by Canadian FASD clinicians

- Find any areas of FASD assessment where adequate assessment measures may be lacking

- Identify additional measures that may be considered beyond those recommended in the current FASD diagnostic guideline

What will we ask you to do? We are asking FASD assessment and diagnostic clinicians (i.e., physicians, psychologists, speech language therapists, and occupational therapists) to complete an online survey. The survey will take approximately 20 minutes to complete. Participation in this study is voluntary and you have the right not to participate. You can discontinue your participation and withdraw from the study at any time without penalty. You may choose to exit the survey by simply closing the browser page. If you choose to discontinue your participation, we will only use the data collected prior to participant withdrawal as it directly relates to the study purpose and procedures.

Are there any risks if I agree to participate? There is an extremely minimal social risk associated with this survey. For example, although no individual-level data will be reported, readers may be able to identify which clinics have participated or which clinics are using particular measures, especially if you work at a small or specialized diagnostic clinic. However, this risk will be minimized by aggregating questionnaire data and no individual clinic information will be available. We believe that this risk is not beyond a risk that you may encounter in your daily practice.

How can you benefit if you agree to participate? There are no direct personal benefits to participation in this study. However, information we gather will provide us with a better understanding of the measures used by clinicians to assess for FASD, and help us to increase consistency, best practices, and sharing of useful information that can benefit other clinics. Ultimately, identifying a comprehensive, reliable, and usable testing battery for FASD assessment will improve the clarity and accuracy of the diagnostic process and facilitate advancements in the field.

How will your information be used? Once the surveys have been completed, we will aggregate the data collected with all identifiers removed. All information we collect will be private and kept confidential. No one will see the answers you give to questions except members of the research team. All information will be stored in a password-protected file. You personal information, such as your clinic name and clinical role, will be kept separate from your survey responses. 
Do you receive anything for participating? All clinics who complete this survey will be entered into a raffle draw for one paid conference registration fee to the 8th International Conference on Fetal Alcohol Spectrum Disorder, March $6-9,2019$, in Vancouver, BC, valued at approximately $\$ 700$.

Do you have questions or want more information? Please contact Kelly Coons-Harding at kharding@laurentian.ca or kelly.harding@canfasd.ca, Colleen Burns at clinictraining@lcfasd.com, or Audrey McFarlane at AMcFarlane@lcfasd.com if you have any questions about this study. If your questions or concerns are not answered, you may contact the Research Ethics Officer, Laurentian University Research Office, at 705-675-1151 or 1-800-461-4030, extention 3213, or at ethics@laurentian.ca.

Consent Statement I understand that: - I do not have to answer all of the questions in the survey - My
answers will be anonymized after they are collected $\square$ Yes All information will be kept confidential and secure - There are no particular risks if I participate - I may withdraw from the study at any time without penalty - I may keep this information about the study I understand that by completing this survey, I am agreeing to participate in this study.

Thank you very much for your time. Have a nice day! 


\section{Clinic Demographic Information}

Your clinic name

Do you provide assessment services at more than one Yes FASD clinic?

If yes: How many FASD clinics do you work with?

If yes: Please list the names, locations (e.g., province or territory), and clinic type (e.g., paediatric, adult, etc.) of all FASD clinics where you provide assessment services.

Do you use the same testing measures at all FASD clinics?

Yes

No

If no: Please describe these differences.

Your clinic type

Paediatric

Adult

Paediatric and Adult

Other

Your province or territory

Alberta

British Columbia

Manitoba

New Brunswick

Nova Scotia

Newfoundland and Labrador

$\square$ Northwest Territories

Nunavut

Ontario

$\square$ Quebec

Saskatchewan

Yukon

Prince Edward Island

What is your profession?

$\square$ Psychologist
$\square$ Family Physician
$\square$ Paediatrician
$\square$ Psychiatrist
$\square$ Nurse or Nurse Practitioner
$\square$ Occupational Therapist
$\square$ Speech Language Pathologist
$\square$ Other

If other, please specify:

Which professions are part of your core diagnostic team? Please check all that apply.

$\square$ Psychologist
$\square$ Family Physician
$\square$ Paediatrician
$\square$ Psychiatrist
$\square$ Nurse or Nurse Practitioner
$\square$ Occupational Therapist
$\square$ Speech Language Pathologist
$\square$ Clinic Coordinator
$\square$ Other

If other, please specify: 
Which additional members are part of your clinical team, where appropriate? Please check all that apply.
Addiction Counsellor Advocate or Mentor Childcare Worker

Clinic Coordinator

Clinical Geneticist

Cultural Interpreter/Liaison

Dysmorphologist

Mental Health Professional

Probation Officer

Social Worker

Teacher

Vocational Counsellor

Other

If other, please specify: 
In the questions below, please indicate which tests you routinely use during your assessment for FASD in each age range. Please indicate only the tests that you actively use in your current assessments. Do not include tests that may be available to you, but that you do not routinely use in your FASD assessments.

If your test does not appear on the list, please enter it in the space below the table ensuring that you indicate which version or edition. Note that the list below includes the most recent edition of each measure. If you use different editions than those listed below, please note your edition in the space below the table.

\section{Motor Skills}

0-3 Months $\quad \begin{gathered}\text { 3-18 Months } \\ \text { Years }\end{gathered}$ 18-36 Months $\begin{gathered}\text { Months }-6 \\ \text { 7-18 Years }\end{gathered}$ 18+ Years

Abnormal Involuntary Movement Scale

Beery-Buktenica Developmental Test of Visual-Motor Integration 6th Ed.

Bruininks-Oseretsky Test of Motor Proficiency - 2nd Ed. Bruininkiks-Oseretsky Test of Motor Proficiency - 2nd Ed. (Complete Form)

Developmental Test of Visual Perception

Finger Tapping/Oscillation Test Grooved/Purdue Pegboard Test Hand Dynamometer/Grip Strength Test

McMaster Handwriting Assessment Protocol

Miller Function \& Participation Scales

Movement Assessment Battery for Children - 2nd Ed.

Peabody Developmental Motor Scales - 2nd Ed.

Quick Neurological Screening Test - 3rd Ed.

Sensory Profile - 2nd Ed.

Sensory Profile Measure

Shore Handwriting Screening

Other

If other, please specify. 
Neuroanatomy/Neurophysiology

0-3 Months

Assessment for physical/medical anomalies associated with FASD (e.g., microcephaly)

History of seizures

Review of past neuroimaging reports

Facial measurements (manual)

Photographic facial analysis software

Other

If other, please specify.
3-18 Months $18-36$ Months 36 Months - $6 \quad$ 7-18 Years Years

$18+$ Years $\square$

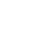




\section{Cognition}

Bayley Scales of Infant and Toddler Development - 3rd Ed.

Detroit Test of Learning Aptitude

Developmental Assessment of Young Children

Mullen Scale of Early Learning

Stanford-Binet Intelligence Scales - 5th Ed.

Wechsler Abbreviated Scale of Intelligence - 2nd Ed.

Wechsler Adult Intelligence Scale - 4th Ed.

Wechsler Intelligence Scale for Children - 5th Ed.

Wechsler Nonverbal Scale of Ability

Wechsler Preschool and Primary Scale of Intelligence - 4th Ed.

Other

If other, please specify. 


\section{Language}

Boston Naming Test - 2nd Ed.

Children's Communication

Checklist - 2nd Ed.

Clinical Evaluation of Language Fundamentals - 5th Ed.

Comprehensive Assessment of Spoken Language - 2nd Ed.

Comprehensive Receptive and Expressive Vocabulary Test - 3rd Edintrolled Oral Word Association Test

Expressive One-Word Picture Vocabulary Test - 4th Ed.

Receptive One-Word Picture Vocabulary Test - 4th Ed.

Expressive Vocabulary Test 2nd Ed.

Listening Comprehension Test

Oral and Written Language

Scales - 2nd Ed.

Peabody Picture Vocabulary Test - 4th Ed.

Preschool Language Scales - 5th Ed.

Receptive-Expressive Emergent Language Test - 3rd Ed.

Renfrew Bus Story

Rosetti Infant Toddler Scale

Social Language Development Test - Elementary: Normative Updates

Social Language Development

Test - Adolescent: Normative

Yesdatê Narrative Language - 2nd

Ed.

Test of Problems Solving - 3rd

Ed. Elementary

Test of Problems Solving - 2nd

Ed. Adolescent

Test of Word Knowledge

Other

If other, please specify. 
Academic Achievement

Bracken Basic Concepts Scale 3rd Ed. Receptive

Process Assessment of the Learner - 2nd Ed.

Wechsler Fundamentals:

Academic Skills

Wechsler Individual

Achievement Test - 3rd Ed.

Wide Range Achievement Test 4th Ed.

Woodcock Johnson IV Tests of Achievement

Other

If other, please specify. 


\section{Memory}

Benton Visual Retention Test

Brief Visuospatial Memory Test Revised

Child and Adolescent Memory Profile

California Verbal Learning Test 2nd Ed.

California Verbal Learning Test Children's

Children's Memory Scale

NEPSY - 2nd Ed.

Rey Auditory Verbal Learning

Reyt Complex Figure Test and Recognition Trial

Rivermead Behavioural Memory Test

Test of Memory and Learning 2nd Ed.

Wechsler Memory Scale - 4th Ed.

Wide Range Assessment of Memory and Learning - 2nd Ed.

Other

If other, please specify. 
Attention

Attention Process Training - 3rd Ed.

Behavior Assessment System for Children - 3rd Ed.

Brief Test of Attention

Conners Continuous

Performance Test - 3rd Ed.

Conners Rating Scales - 3rd Ed.

Conners Adult ADHD Rating

Scales

Digital Vigilance Test

Integrated Visual and Auditory Learning - 2nd Ed.

NEPSY - 2nd Ed.

Spatial Span

Swanson Nolan and Pelham

Questionnaire - 4th Ed.

Visual Search and Attention Test

Other

If other, please specify. 


\section{Executive Function, including Impulse Control and Hyperactivity}

0-3 Months $\quad \begin{gathered}\text { 3-18 Months } \\ \text { Years }\end{gathered}$ 18-36 Months $\begin{gathered}\text { Months }-6 \\ \text { 7-18 Years }\end{gathered}$ 18+ Years

Behavior Rating Inventory of

Executive Function - 2nd Ed.

Behavior Rating Inventory of

Executive Function - Adult

Brixton Spatial Anticipation Test

Category Test (Booklet,

Children's, or Halstead)

Comprehensive Executive

Function Inventory

Comprehensive Executive

Function Inventory - Adult

Delis-Kaplan Executive Function System

Functional Assessment of Verbal

Reasoning and Executive

Strategies

lowa Gambling Task

NEPSY - 2nd Ed.

Rey Complex Figure Test and

Recognition Trial

Rey-Osterreich Complex Figure

Social Language Development Test - Elementary: Normative Updates

Social Language Development Test - Adolescent: Normative Stroop Test

Symbol Digit Modalities Test

Test of Problems Solving - 3rd

Ed. Elementary

Test of Problems Solving - 2nd

Ed. Adolescent

Trail Making Test

Wisconsin Card Sort Task

Other

If other, please specify. 


\section{Affect Regulation}

Beck Anxiety Inventory

Beck Depression Inventory - 2nd Ed.

Beck Youth Inventory - 2nd Ed.

Behavior Assessment System for Children - 3rd Ed.

Behavior Dimensions Scale - 2nd Ed.

Child Behavior Checklist

Millon Adolescent Clinical Inventory

Millon Clinical Multiaxial Inventory - 4th Ed.

Mini International Personality Item Pool

Minnesota Multiphasic

Personality Inventory - 2nd Ed.

Minnesota Multiphasic

Personality Inventory -

Pdolescrent Assessment

persontory Yty Assessment Inventory - Adolescent

Screen for Child Anxiety Related Disorders

Symptom Checklist-90-Revised

Trauma Symptom Inventory 2nd Ed.

Other

If other, please specify. 
Adaptive Behaviour, Social Skills, and Social Communication
0-3 Months
3-18 Months
18-36 Months
36 Months - 6
7-18 Years
$18+$ Years

Adaptive Behavior Assessment System - 3rd Ed.

Advanced Clinical Solutions for the WAIS-IV and WMS-IV

Behavior Assessment System for Children - 3rd Ed.

Clinical Evaluation of Language Fundamentals - 5th Ed.

Independent Living Scales

Scales of Independent Behavior Revised

Social Language Development

Test - Elementary: Normative Updates

Social Language Development Test - Adolescent: Normative Ypdates Awareness of Social Interference Test - Revised

Test of Social Language Development

Texas Functional Living Scales

Vineland Adaptive Behavior

Scales - 2nd Ed.

Other

If other, please specify.

Thank you very much for taking the time to complete this survey. Have a nice day! 